\title{
Negative-Pressure Wound Therapy for a Replanted Finger with Complicated Wound
}

\author{
Si Young Roh (D), llou Park (D), Kyung Jin Lee (D), Sung Hoon Koh (D), Jin Soo Kim (D) \\ Department of Plastic and Reconstructive Surgery, Gwangmyeong Sungae Hospital, Gwangmyeong, Korea
}

\begin{abstract}
Negative-pressure wound therapy (NPWT) is widely used for open wounds in various anatomical sites. Extensive research has been carried out on the application of NPWT; exposed blood vessels in the periwound area are a known contraindication to NPWT. In this study, we report a case where a replanted finger with not only exposed vessels but also uncontrolled infection was treated with NPWT. A 60-year-old man visited our emergency department with incomplete amputation of his left index and middle fingers. After replantation of his two fingers, necrosis of the middle finger with severe methicillin-resistant Staphylococcus aureus infection complicated managing the wound. After 3 weeks of maintaining conventional wound dressings, we performed NPWT for successful granulation tissue formation and infection control. After the treatment, the wound was completely covered by a split-thickness skin graft. We thus suggest that NPWT can be an effective reconstructive method, including for intractable wounds with exposed pedicle and severe infection after replantation.
\end{abstract}

Keywords: Negative-pressure wound therapy; Replantation; Hand reconstruction; Infection

\section{Introduction}

Negative-pressure wound therapy (NPWT) is widely used for a range of acute and chronic wounds [1]. NPWT helps wound healing by maintaining moisture, removing exudate, increasing local vascularity, decreasing bacterial colonization, stimulating granulation tissue, and so on [2].

However, it is contraindicated in cases with malignancy, necrosis, fibrosis, exposed vessels, and uncontrolled infection [3]. Moreover, indications of NPWT have been varied, whereas contraindications of NPWT are still controversial [4]. For this reason, deciding on a therapeutic option is often challenging in cases of contraindications.

We hereby report our experience with a case where NPWT was used to treat a replanted finger with exposed blood vessels and severe infection, which are contraindications to NPWT, and discuss precautions for such treatment.

The study was approved by the Institutional Review Board of Gwangmyeong Sungae Hospital (IRB No. KIRB-2020-N-005) and performed in accordance with the principles of the Declaration of Helsinki. The patient provided written informed consent for the publication and the use of his images.

\section{Case}

A 60-year-old man with amputated fingers after an injury caused by a press machine visited our emergency department. We immediately performed replantation of his left index and middle fingers (Fig. 1A). Whereas both digital arteries of the amputated index finger could be directly anastomosed, the ulnar and radial digital arteries of
Case Report

Received: October 5, 2020

Revised: January 19, 2021

Accepted: January 20, 2021

\section{Corresponding author:}

Kyung Jin Lee, M.D.

Department of Plastic and Reconstructive Surgery, Gwangmyeong Sungae Hospital, 36 Digital-ro, Gwangmyeong 14241, Korea Tel: +82-2-2680-7637

Fax: +82-2-2615-7218

E-mail: telotism@naver.com

This is an Open Access article distributed under the terms of the Creative Commons Attribution Non-Commercial License (https://creativecommons.org/licenses/by-nc/4.0/) which permits unrestricted non-commercial use, distribution, and reproduction in any medium, provided the original work is properly cited.

(c) 2021 Korean Wound Management Society 

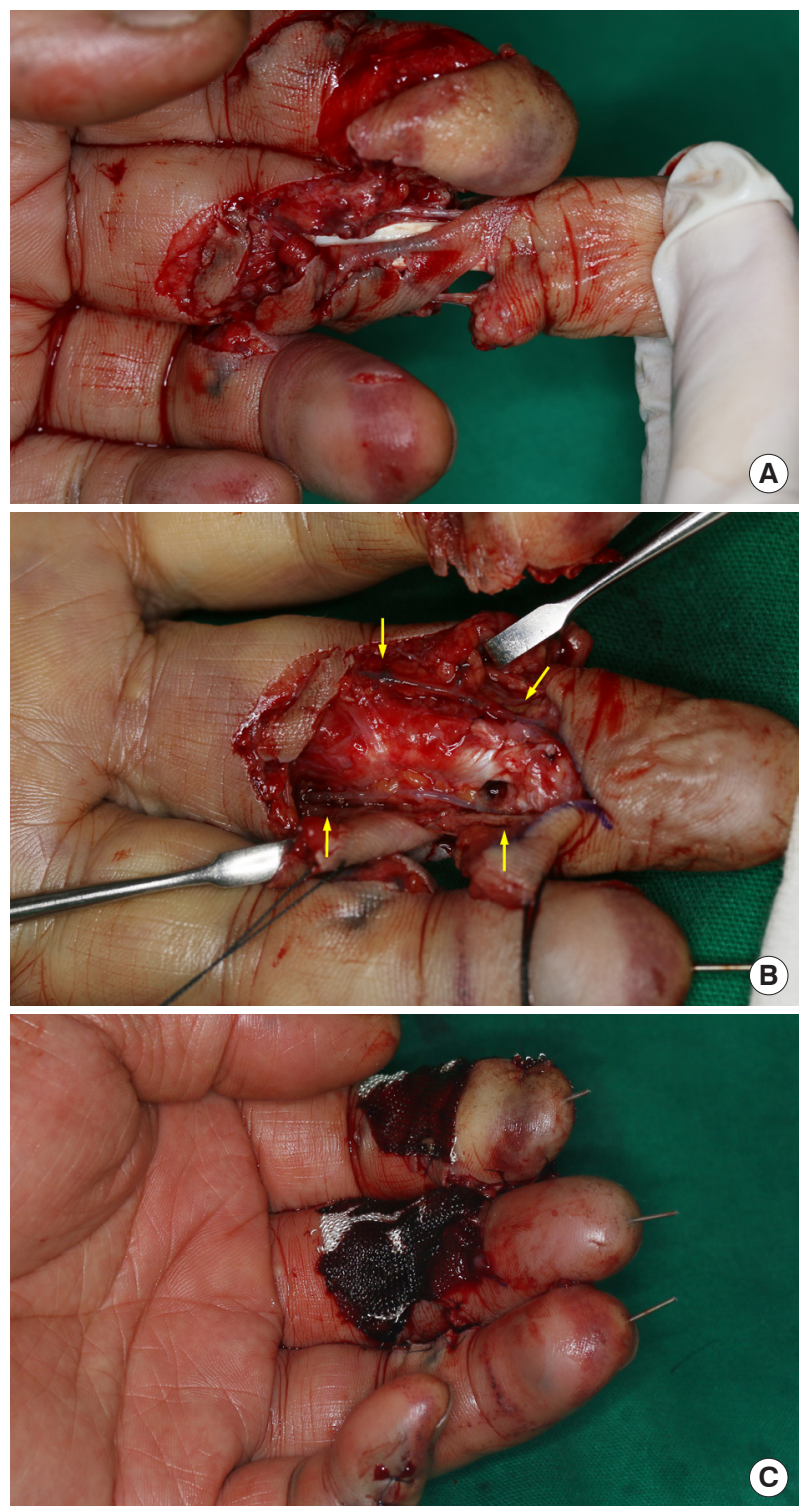

Fig. 1. Preoperative, intraoperative, and postoperative photographs. (A) A 60-year-old man was injured by a press machine that caused incomplete amputation of his left index and middle fingers. (B) The digital arteries of the middle finger were anastomosed using vein grafts (yellow arrows). (C) The index and middle fingers were replanted.

his middle finger could be anastomosed only with vein grafts, owing to thrombi of both digital arteries (Fig. 1B and C). Although the patient's two replanted fingers survived, necrosis and methicillin-resistant Staphylococcus aureus (MRSA) infection in the middle finger was noted through wound culture on postoperative day 10. The complex wound showed little improvement even after 2 weeks of intravenous injection of vancomycin ( $1 \mathrm{~g}$ twice a day). Due to his active infection, the patient was not in a state to receive another flap surgery to

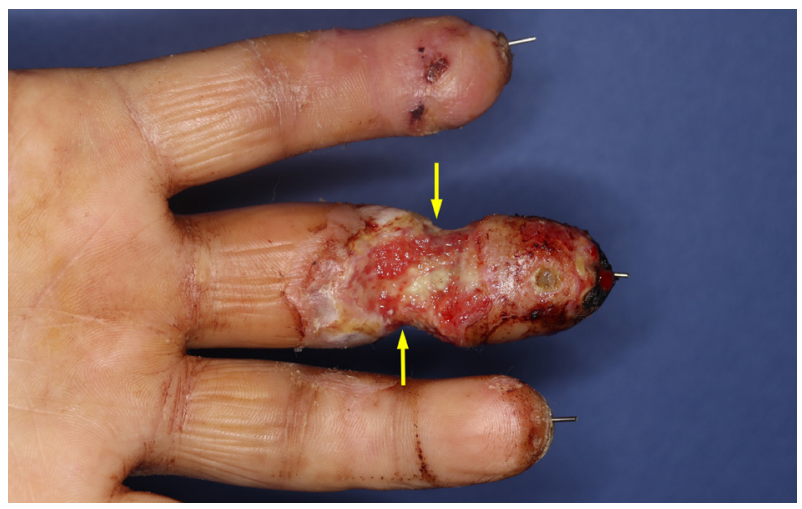

Fig. 2. The replanted middle finger with a complex wound. A soft tissue defect with severe infection and exposed digital vessels (yellow arrows) are shown.

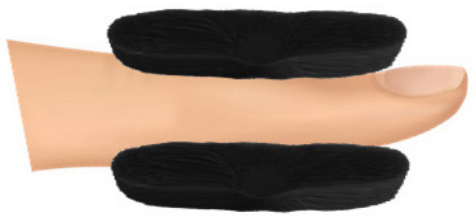

(Lateral view)

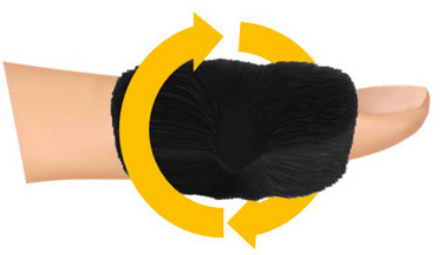

(Lateral view)

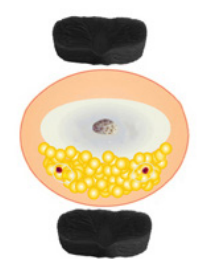

(Axial view)

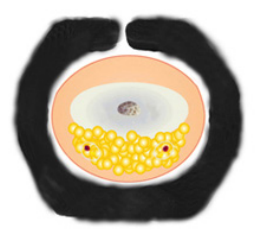

(Axial view)
Fig. 3. Schematic of negative-pressure wound therapy sponge modification. (A) The sponge is open laterally-toward ulnar and radial side-so that the sponge does not directly compress the vascular area. (B) The wound is sealed with the sponge circumferentially in the third cycle.

cover the wound. Since previous replantation had been done with vein grafts for both digital arteries of the middle finger, the finger could not provide stable arterial blood stream for additional flap coverage.

Therefore, even though the wound was intractable to infection control measures (Fig. 2), we decided to conserve the replanted finger and apply negative pressure of $-75 \mathrm{mmHg}$ at an intermittent mode (5-minute suction and 2-minute rest) to prevent ischemic change, for the first 3-day cycle of NPWT, using an InfoV.A.C. Therapy Unit (KCI Medical, Inc., Mississauga, ON, Canada) after debridement. The wound was sealed with a NPWT sponge, opened laterally to minimize the exposure of the vessels (Fig. 3A). The blood circulation in the re- 
planted finger was maintained for the first cycle, and granulation tissue began to form and cover both pedicles (Fig. 4A). We increased the negative pressure up to $-100 \mathrm{mmHg}$ for the second cycle, keeping the same intermittent mode and sealing
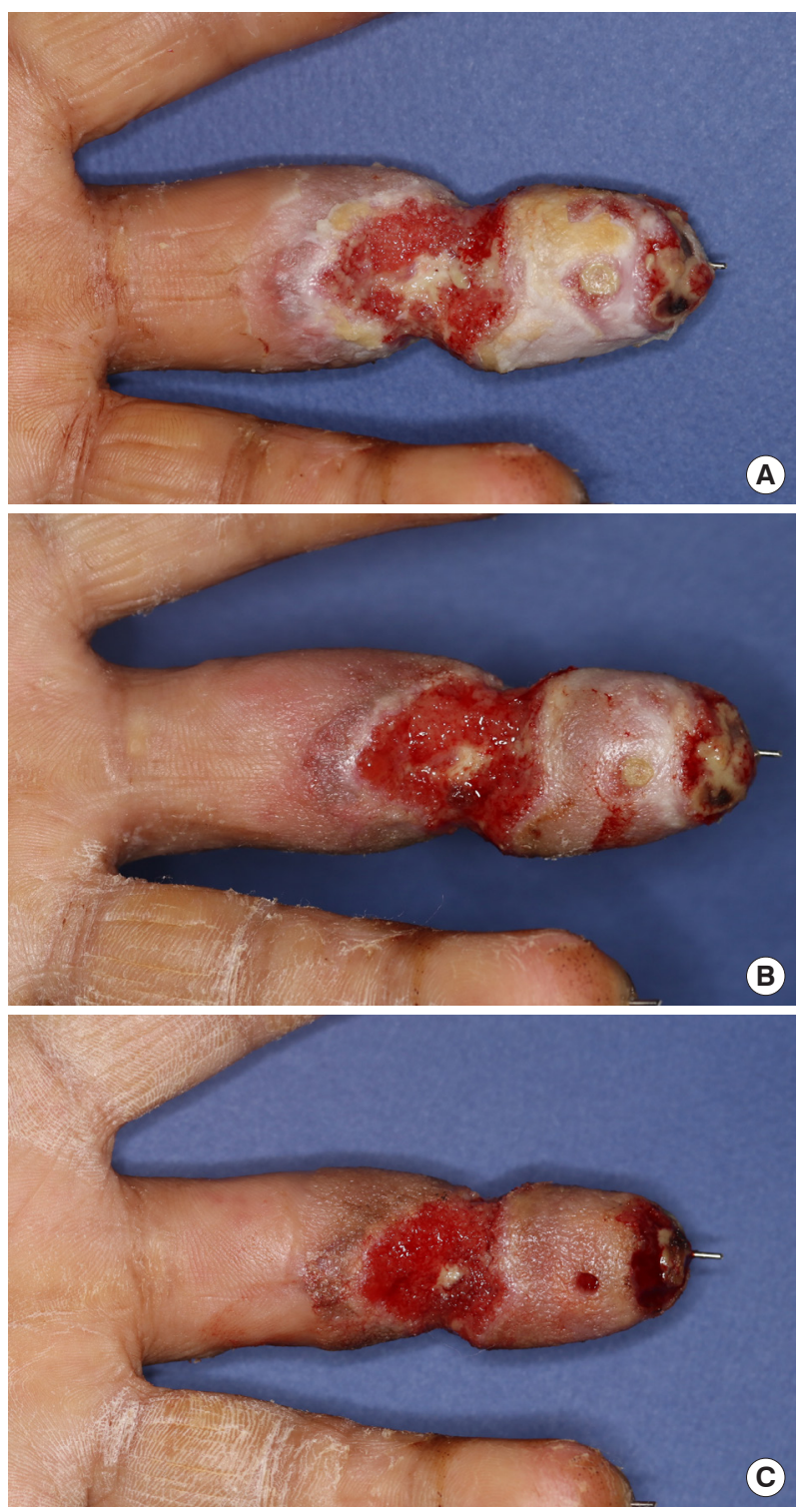

Fig. 4. Applying negative-pressure wound therapy on the replanted finger. (A) After the first cycle. (B) After the second cycle. (C) After the third cycle. technique with the sponge opened laterally, as in the previous cycle (Table 1, Fig. 4B). In the third cycle, we sealed the finger circumferentially since both vessels were fully covered (Table 1, Fig. 3B). After three cycles of NPWT, the granulation tissue fully covered the exposed digital vessels (Fig. 4C) and signs of infection such as erythema, odor, and discharge decreased. Inflammatory markers such as erythrocyte sedimentation rate and C-reactive protein level decreased from $45 \mathrm{~mm} / \mathrm{hr}$ and $3.82 \mathrm{mg} / \mathrm{dL}$ respectively on postoperative day 10 , to their normal ranges on postoperative day 27 . On postoperative day 43 , the final follow-up wound culture confirmed the absence of bacterial growth. Finally, we covered the wound with only a split-thickness graft and discharged the patient on postoperative day 66 .

The patient's replanted left middle finger survived well without any circulatory complications and maintained full coverage and intact blood circulation, as confirmed in tracings with a handheld Doppler ultrasonographic device at 2 and 4 months after discharge (Fig. 5). Our primary goal was to cover the wound as soon as possible, and the contracture caused by split-thickness skin graft on distal phalangeal joint had been expected. Indeed, this patient had tenolysis surgery of the finger and improved range of motion of the replanted finger 3 years after discharge (Fig. 6).

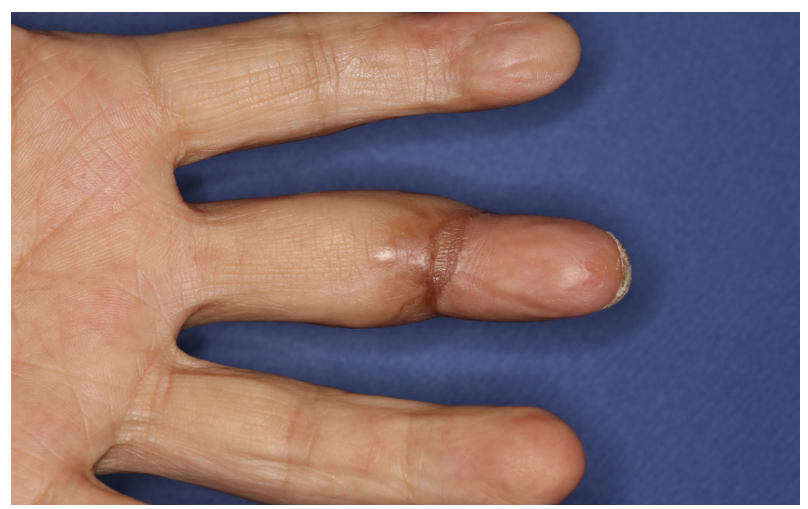

Fig. 5. Long term follow-up photograph. Four months after discharge, the patient's replanted middle finger maintained good full coverage.

Table 1. Modifications of negative-pressure wound therapy application

\begin{tabular}{llcc}
\hline & Mode & Pressure (mmHg) & Sealing type \\
\hline 1st cycle & Intermittent (5-minute suction \& 2-minute rest) & -75 & Open laterally \\
2nd cycle & Intermittent (5-minute suction \& 2-minute rest) & -100 & Open laterally \\
3rd cycle & Intermittent (5-minute suction \& 2-minute rest) & -100 & Circumferential \\
\hline
\end{tabular}



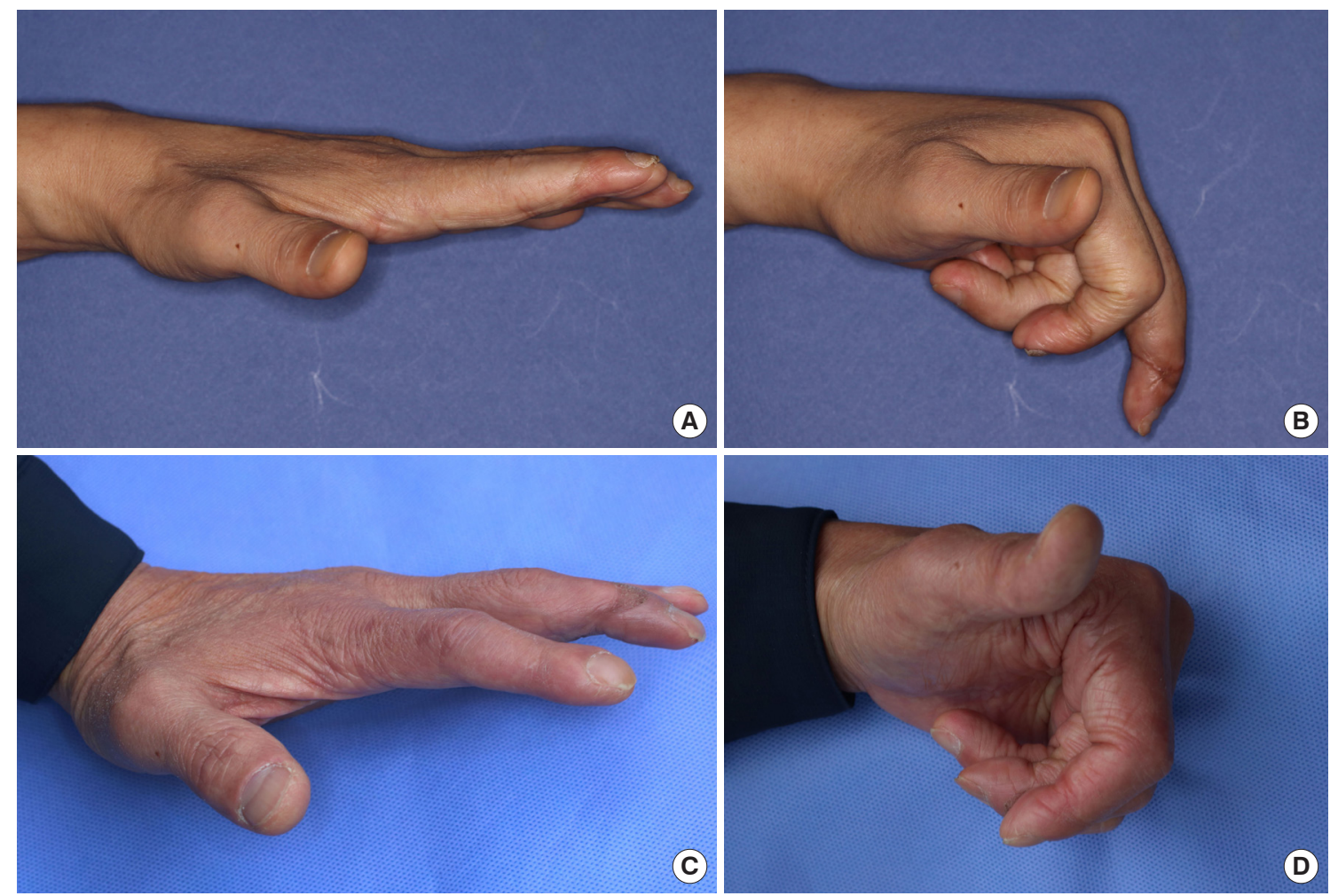

Fig. 6. Improved range of motion of the middle finger. (A, B) Preoperative photograph of tenolysis. (C, D) Three years follow-up photograph after tenolysis.

\section{Discussion}

NPWT is widely known to be useful for managing various types of wounds at different anatomical sites. It has been recently modified with a variety of applications and methods [1]. It is thought to help promote wound healing through several mechanisms such as macrodeformation, microdeformation, fluid removal, and optimization of the wound environment. NPWT thus plays a significant role in managing chronic, complex, and infected wounds [1,2].

Although some secondary procedures after replantation have been studied, the use of NPWT in such cases has not yet been reported $[5,6]$. NPWT is also not recommended for managing wounds with MRSA infection [7].

In this case, application of NPWT for a complex wound with exposed vessels and uncontrolled infection had been disputable. However, the condition of the replanted finger was not suitable for coverage with a free flap and vein graft or a regional flap, because the adjacent index and ring fingers were crushed. In addition, without immediate coverage, all portions of the replanted finger would have had necrosis.

Although no cases using NPWT to treat wounds of replanted fingers have been reported, we nevertheless considered NPWT as a means to conserve the replantation, and needed an appropriate modulation for applying NPWT to the complex wound. To maintain blood flow, the negative pressure applied was not to exceed the average finger blood pressure $(110 \mathrm{mmHg})$, and the NPWT sponge was to be used for sealing only on the volar and dorsal sides of the wound in the first NPWT cycle, to prevent choking the wound with exposed vessels [8]. The sponge could then be sealed circumferentially in the second and third cycles, after checking that granulation tissue had covered the wound pedicles, and using a handheld Doppler ultrasonographic device to confirm the blood flow was intact.

In general, the negative pressure applied in NPWT ranges from -125 to $-175 \mathrm{mmHg}$, in intermittent or continuous mode. A negative pressure of $-125 \mathrm{mmHg}$ is the baseline setting for all kinds of wounds. The effect of the intermittent mode (5-minute suction and 2-minute rest) on wound healing is equivalent to that of the continuous mode [9]. Moreover, as granulation tissue formation takes place in the intermittent mode as much as in continuous NPWT mode, the NPWT mode should be tailored to individual wounds for optimal effects [10]. According to Wackenfors et al. [11], the effective 
negative pressures for minimizing possible ischemic effects were $-75 \mathrm{mmHg}$ for soft tissue and $-100 \mathrm{mmHg}$ for muscle tissue. Accordingly, we applied a $-75 \mathrm{mmHg}$ pressure for the first cycle and increased it to $-100 \mathrm{mmHg}$ for the second and third cycles, maintaining the intermittent mode.

Even though NPWT is being applied for an increasingly broader range of wounds, anatomical sites, and medical conditions, whether the replanted finger in this case can be considered a definite indication for NPWT might not be affirmed. Nevertheless, we demonstrated that a wound with vascular exposure and active MRSA infection could be safely managed with NPWT. We therefore conclude that NPWT could be a possible option for replantation with intractable wounds under appropriate negative pressure modulations.

\section{Conflict of interest}

No potential conflict of interest relevant to this article was reported.

\section{ORCID iDs}

Si Young Roh

Ilou Park

Kyung Jin Lee

Sung Hoon Koh

Jin Soo Kim

\section{References}

1. Anghel EL, Kim PJ. Negative-pressure wound therapy: a comprehensive review of the evidence. Plast Reconstr Surg
2016;138(3 Suppl):129S-137S.

2. Huang C, Leavitt T, Bayer LR, et al. Effect of negative pressure wound therapy on wound healing. Curr Probl Surg 2014;51:301-31.

3. National Health Service (NHS). Guideline for the use of negative pressure wound therapy (NPWT). Leeds: National Health Service (UK); 2017.

4. Mendez-Eastman S. Guidelines for using negative pressure wound therapy. Adv Skin Wound Care 2001;14:314-22.

5. Chung KC, Alderman AK. Replantation of the upper extremity: indications and outcomes. J Am Soc Surg Hand 2002;2:78-94.

6. Sabapathy SR, Bhardwaj P. Secondary procedures in replantation. Semin Plast Surg 2013;27:198-204.

7. Han SE, Hwang SO, Lee SH, et al. Skin and soft tissue infections in plastic surgery over 10 years. J Wound Manag Res 2019;15:11-6.

8. Welsh AJ, Griffin MJ. Normal values for finger systolic blood pressures in males and females. Int Arch Occup Environ Health 2008;81:625-32.

9. Morykwas MJ, Argenta LC, Shelton-Brown EI, et al. Vacuum-assisted closure: a new method for wound control and treatment: animal studies and basic foundation. Ann Plast Surg 1997;38:553-62.

10. Malmsjo M, Gustafsson L, Lindstedt S, et al. The effects of variable, intermittent, and continuous negative pressure wound therapy, using foam or gauze, on wound contraction, granulation tissue formation, and ingrowth into the wound filler. Eplasty 2012;12:e5.

11. Wackenfors A, Sjogren J, Gustafsson R, et al. Effects of vacuum-assisted closure therapy on inguinal wound edge microvascular blood flow. Wound Repair Regen 2004;12:600-6. 